\title{
Intracranial Major Artery and Venous Sinus Thrombosis in a Young Male with MTHFR Mutation and Protein S Deficiency
}

Dhanashree Peddawad

Jupiter Hospital, Pune, India

Keywords

Young stroke - Methylenetetrahydrofolate reductase mutation - Protein S deficiency .

Arterial and venous thrombosis

\section{Abstract}

This case represents a unique example of stroke in a young patient involving major venous sinuses as well as major artery in a span of 6 months. After evaluation, he was found to have an abnormal thrombophilia profile. In young patients with recurrent stroke, investigating for an abnormal thrombophilia profile is crucial.

\section{Introduction}

This case represents a unique example of stroke in a young patient involving major venous sinuses as well as major artery in a span of 6 months. After evaluation, he was found to have an abnormal thrombophilia profile. In young patients with recurrent stroke, investigating for an abnormal thrombophilia profile is crucial.

\section{Patient Information}

A 32-year-old male patient was evaluated for recurrent stroke within a span of 6 months. $\mathrm{He}$ is an alcoholic and smoker for 6 years. 


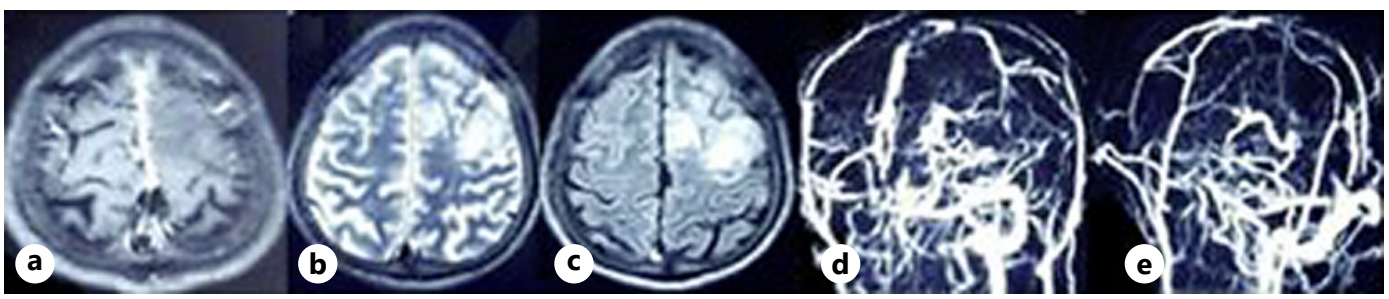

Fig. 1. a T1 C axial - left high frontal hypointensity and filling defect in SSS. b, c T2 axial and FLAIR axial - left frontal hyperintensities. d, e Three-dimensional reconstructed images of contrast venography - right transverse, sigmoid, and SSS filling defect. SSS, superior sagittal sinus.

\section{Clinical Findings and Timeline}

The patient presented with headache of 5-day duration and right-sided weakness for 1 day. There were no fever, vomiting, diarrhea, or recent trauma. He had a pulse of 90 per minute and regular rhythm and blood pressure 140/80 mm Hg. On neurological examination, he was conscious oriented and had right hemiparesis with right facial palsy. His routine blood tests like complete blood count, liver function, renal function tests, lipid profile, and fasting and postprandial blood sugar were normal. Electrocardiogram was suggestive of sinus rhythm, and 2D echo and X-ray chest were normal. He underwent MRI brain which showed 2 patchy areas of the left high frontal acute infarcts (Fig. 1). Considering the patient's age, symptoms, and location of infarcts, he underwent contrast venography, which revealed thrombosis of the superior sagittal sinus, right transverse, sigmoid sinus, and right internal jugular vein. Contrast venography revealed filling defect in almost all right-sided major venous sinuses. In view of the above findings, the patient was given heparin followed by oral anticoagulation. Hemiparesis recovered within few weeks.

Five months later, he developed left hemiplegia with dysarthria. Initially, he also had leftsided hemineglect which recovered within 1 week. He underwent CT brain which showed acute infarct involving the right MCA territory. MR angiography showed right ICA and right MCA complete thrombosis (Fig. 2). Antiplatelets were prescribed. Warfarin was continued. There was partial recovery with treatment and physiotherapy. But after 1 month, he developed severe headache, vomiting, and increase in existing left-sided weakness. He was drowsy but obeying commands. CT brain revealed hemorrhagic transformation of the right MCA infarct with midline shift of $1 \mathrm{~cm}$. All blood thinners were stopped. He received antiedema treatment. During the hospital course, his sensorium improved and headache subsided. He was prescribed vitamin B12 supplement, antiepileptic, and lipid-lowering medication.

\section{Diagnostic Assessment}

Specific blood investigations were sent in a follow-up visit at 4 weeks (Table 1 ; thrombophilia profile). Anti-nuclear antibody profile, p-anti-neutrophil cytoplasmic antibodies, and c-anti-neutrophil cytoplasmic antibodies were negative. Serum homocysteine was normal. The thrombophilia profile was sent: protein S activity, protein C activity, antithrombin III activity, APCR-activated protein C resistance, lupus anticoagulant profile, cardiolipin antibody ACL-IgG, cardiolipin antibody ACL-IgM, factor V mutation, methylenetetrahydrofolate reductase (MTHFR) gene mutation, and factor II (prothrombin) mutation. Amongst all the above tests, protein $S$ activity was low with a low value of protein $S$ antigen (free) as well. Also, MTHFR mutations, (C677T) and (A1298C), were detected. 


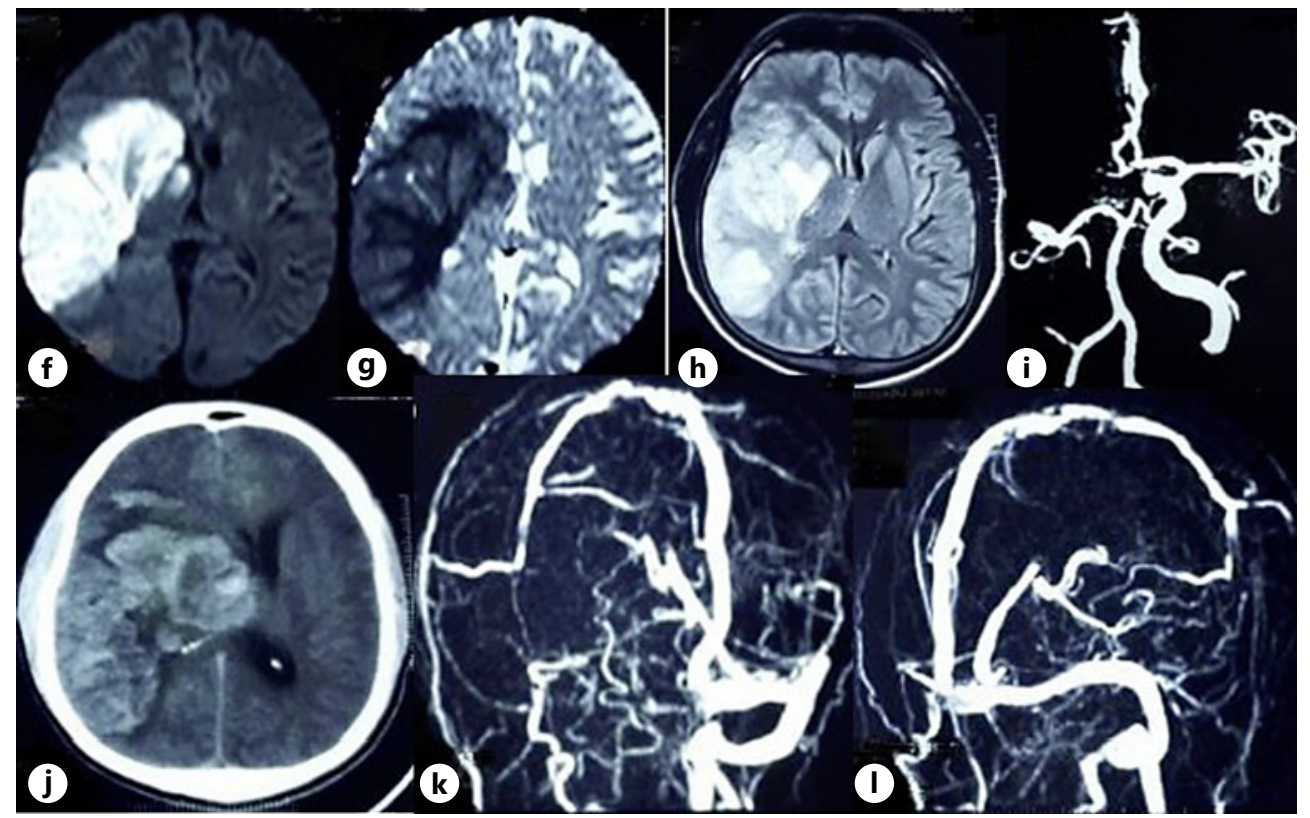

Fig. 2. a DWI, b ADC, and c FLAIR axial - right MCA acute infarct. d MRAngio-right ICA and right MCA showing complete thrombosis. e CT axial section - hemorrhagic transformation with midline shift. $\mathbf{f}, \mathbf{g}$ Follow-up MR venography shows partial recanalization of SSS but persistent right transverse and sigmoid sinus thrombosis.

\section{Therapeutic Information}

For the first stroke due to venous sinus thrombosis, he received heparin followed by warfarin. He was also prescribed antiepileptic levetiracetam. In view of the right MCA infarct, aspirin and clopidogrel $75 \mathrm{mg}$ each were prescribed. Due to hemorrhagic transformation, warfarin, aspirin, and clopidogrel were stopped for 6 weeks. Then, blood thinners were introduced one by one. Initially, aspirin $75 \mathrm{mg}$ was added, which was increased to $150 \mathrm{mg}$ after 4 weeks. Dabigatran was added 8 weeks from the introduction of aspirin. As the patient was not keen on doing regular PT INR monitoring, dabigatran was added which does not require INR testing. He also received levetiracetam and atorvastatin.

\section{Follow-Up and Outcome}

Gradually symptomatic as well as clinical improvement was noted. Six months after the second stroke, the left upper and lower limb power was 3/5.

\section{Discussions}

It is very unusual to have intracranial arterial as well as venous stroke that too involving major arteries and major venous sinuses. The patient is an example of a very rare case with both intracranial major arterial as well as major venous sinuses thrombosis.

It has been discussed that venous and arterial thromboses are different manifestations of the same disease. As there are some common risk factors for both arterial and venous thromboses, it is possible that both types share a common underlying pathophysiology [1-3]. 
Table 1. Thrombophilia profile of the patient

\begin{tabular}{lll}
\hline Test name & Observed value & Normal range \\
\hline Protein S activity, \% & $\mathbf{2 3}$ & $77-143$ \\
Protein S antigen (free) & $\mathbf{8 7}$ & $89.5-128.5$ \\
(citrated plasma, immunoturbidiometry), \% & & \\
Protein C activity, \% & 98 & $70-130$ \\
Antithrombin III activity, \% & 112 & $80-120$ \\
APCR-activated protein C resistance & $156.2 \mathrm{~s}$ & $<0 \mathrm{R}=120$ \\
Lupus anticoagulant & Absent & Absent \\
aPTT (test) & $31.1 \mathrm{~s}$ & $35.08-43.81$ \\
DRVV screen (test) & $37.3 \mathrm{~s}$ & $32.50-45.86$ \\
Cardiolipin antibody ACL - IgG (serum, EIA) & Negative $(3.58)$ & Negative: $<10$ \\
& GPL U/mL & Positive: $\geq 10$ \\
Cardiolipin antibody ACL - IgM (serum, EIA) & Negative $(2.98)$ & Negative: $<7$ \\
& MPL U/mL & Positive: $\geq 7$ \\
PR3 - ANCA (C-ANCA) ELISA & $3.5 \mathrm{U} / \mathrm{mL}$ & $<6 \mathrm{U} / \mathrm{mL}$ \\
MPO - ANCA (P-ANCA) ELISA & $2.36 \mathrm{U} / \mathrm{mL}$ & $<12.0 \mathrm{U} / \mathrm{mL}$ \\
Anti-phospholipid antibody - IgG ELISA & $2.72 \mathrm{U} / \mathrm{mL}$ & $<12$ \\
Anti-phospholipid antibody - IgM ELISA & $2.97 \mathrm{U} / \mathrm{mL}$ & $<12$ \\
Homocysteine photometry & $11.7 \mu \mathrm{mol} / \mathrm{L}$ & $<30$ \\
ANA profile & Negative & \\
Factor V Leiden mutation & Not detected & \\
Factor II (prothrombin) mutation & Not detected & \\
Methylenetetrahydrofolate reductase, mutation (C677T) & Detected & \\
Methylenetetrahydrofolate reductase, mutation (A1298C) & Detected & \\
\hline
\end{tabular}

It has also been observed that patients with venous thromboembolism have increased risk of arterial strokes [4].

In this case, the patient is young and had major intracranial artery thrombosis which is unusual at this age. Arterial thrombosis occurred only 5 months after venous thrombosis. The patient was on oral anticoagulant while he developed arterial stroke. Although venous sinus thrombosis can be explained by presence of MTHFR mutation and protein S deficiency, it is possible that these abnormalities could have played an important role in developing major artery thrombosis as well. There are few reports in the literature which have shown association of MTHFR mutation and protein S deficiency as an independent risk factor for arterial stroke. And, this association has been observed mainly in young arterial strokes.

According to previous studies, it has been suggested that MTHFR is associated with young arterial strokes [5]. Homozygotes are found to have more risk compared to heterozygotes in MTHFR 677T mutation [6]. According to another study, MTHFR mutation combined with other genetic mutations is associated with arterial stroke in the young [7]. Heterozygote mutation is also associated with recurrent arterial stroke [8].

Patients with protein $\mathrm{S}$ deficiency are at higher risk of arterial thromboembolism below 55 years of age $[9,10]$. In 1 case, protein $S$ deficiency was associated with arterial stroke followed by pulmonary thromboembolism [11]. Protein $S$ deficiency has also been described with recurrent ischemic stroke [12]. Thus, it is important to find out the etiology of stroke in young patients. 
The patient was consuming alcohol intermittently in moderate quantity since 6 years. He used to smoke about 5-10 cigarettes per day since 6 years. In addition to abnormal thrombophilia, alcohol and smoking could have played a role in the formation of the thrombus.

\section{Statement of Ethics}

The report is exempt from ethics committee approval because this study is a case report and did not require any new intervention. Written informed consent was obtained from the patient for publication of this case report and any accompanying images.

\section{Conflict of Interest Statement}

The author has no conflicts of interest to declare.

\section{Funding Sources}

This case study did not require any funding source.

\section{Data Availability Statement}

All data generated or analyzed during this study are included in this article.

\section{References}

1 Prandoni P. Venous and arterial thrombosis: two aspects of the same disease? Eur J Intern Med. 2009;20(6): 660-1.

2 Lowe GD. Common risk factors for both arterial and venous thrombosis. Br J Haematol. 2008;140:488-95.

3 Prandoni P, Bilora F, Marchiori A, Bernardi E, Petrobelli F, Lensing AW, et al. An association between atherosclerosis and venous thrombosis. N Engl J Med. 2003;348:1435-41.

4 Sorensen HT, Horvath-Puho E, Pedersen L, Baron JA, Prandoni P. Venous thromboembolism and subsequent hospitalisation due to acute arterial cardiovascular events: a 20-year cohort study. Lancet. 2007;370:1773-9.

5 Alluri RV, Mohan V, Komandur S, Chawda K, Chaudhuri JR, Hasan Q. MTHFR C677T gene mutation as a risk factor for arterial stroke: a hospital based study. Eur J Neurol. 2005;12:40-4.

6 Cronin S, Furie KL, Kelly PJ. Dose-related association of MTHFR 677T allele with risk of ischemic stroke: evidence from a cumulative meta-analysis. Stroke. 2005;36:1581-7.

7 Araji AA, Sawaya HR, Sawaya RA. Gene mutations and stroke in the young adult. J Stroke Cerebrovasc Dis. 2014 Nov-Dec;23(10):2554-8.

8 Panigrahi I, Chatterjee T, Biswas A, Behari M, Choudhry PV, Saxena R. Role of MTHFR C677T polymorphism in ischemic stroke. Neurol India. 2006 Mar;54(1):48-2.

9 Mahmoodi BK, Brouwer JL, Veeger NJ, van der Meer J. Hereditary deficiency of protein C or protein S confers increased risk of arterial thromboembolic events at a young age: results from a large family cohort study. Circulation. 2008;118(16):1659-67.

10 Girolami A, Simioni P, Lazzaro AR, Cordiano I. Severe arterial cerebral thrombosis in a patient with protein S deficiency (moderately reduced total and markedly reduced free protein S): a family study. Thromb Haemost. 1989;61:144-7.

11 Wagh SB, Anadure R, Dutta V, Sandhu MS, Trehan R. Isolated protein S deficiency presenting as catastrophic systemic arterial and subsequently venous thrombosis. Australas Med J. 2012;5(8):424-8.

12 Hooda A, Khandelwal PD, Saxena P. Protein S deficiency: recurrent ischemic stroke in young. Ann Indian Acad Neurol. 2009;12(3):183-4. 